\title{
Bilan énergétique et gaz à effet de serre : perspectives agricoles
}

Raymond REAU

Cétiom,

INRA UMR Agronomie, BP1,

78850 Thiverval Grignon

$<$ Raymond.Reau@grignon.inra.fr>

\begin{abstract}
According to an official french life cycle study, the energetic balance of biodiesel produced from oil seed crops is about 2.99 for oil seed rape and 3.16 for sunflower. Diesel greenhouse gas emissions are roughly four times as important as biodiesel emissions from oil seed crops. So presently, these two major performances of sustainability result very positive. For oil seed rape, development of acreage on deep soils, decrease of $N$ fertilisation rate, decrease of energetic costs in $N$ fertilisers industry and development of minimum tillage should continue to improve on energetic balance. On the contrary, for sunflower, the present trend is not an improvement of its energetic balance. In order to go more ahead, more abrupt changes are needed: as reduction of $\mathrm{N}$ applications for sunflower, and oil seed rape plant breeding in order to increase $N$ content of seeds and decrease $N$ content of its harvest residues.
\end{abstract}

Key words: oil seed crops, biodiesel, energetic balance, greenhouse gas de biodiesel est respectivement de 3,0 et 3,2: autrement dit, ces filières produisent en énergie trois fois ce qu'elles consomment (tableau 1). Ce rendement énergétique progresse de 0,9 à 3 quand on passe de la filière gazole à la filière EMVH.

Ces résultats fondés sur des estimations effectuées en 2002 concernaient les perspectives d'évolution 2005, c'est-à-dire celles correspondant concrètement à la campagne que l'on vient de vivre. La réalité actuelle correspondelle à la réalité des pratiques actuelles ?

Si on analyse la situation, aux travers des enquêtes effectuées par le Cetiom on obtient le tableau suivant (tableau 2) présentant la médiane du rendement énergétique du colza Diester $^{\circledR}$ à l'échelle de la France de 1996 à 2005 telle que nous avons pu l'estimer, compte tenu des pratiques agricoles.

Les deux lignes du tableau encadrant cette valeur médiane présentent les résultats pour les premiers et dernier quintile (valeurs dépassées dans 1 cas sur 5). Le ratio énergétique (énergie restituée/énergie non renouvelable mobilisée) de la production certain nombre d'avantages liés à la filière biodiesel obtenu à partir de colza et tournesol. duction de biodiesel : le colza et, plus récemment, le tournesol dont l'huile est transformée

Tableau 1. Bilan énergétique des filières biocardurants complètes en 2005 (huile végétale/ester et gazole). Rendement : énergie restituée/énergie non renouvelable mobilisée.

\begin{tabular}{|lcccc|}
\hline Gazole & Huile de colza & Huile de tournesol & EMHV colza & EMHV tournesol \\
\hline 0,917 & 4,68 & 5,48 & 2,99 & 3,16 \\
\hline
\end{tabular}

EMHV : ester méthylique d'huile végétale.
Entre ces deux quintiles, on constate une variabilité de ce rendement énergétique dans une amplitude de $20 \%$.

La variabilité de cette moyenne en fonction des régions de production (tableau 3 ) montre que des régions ont des rendements énergétiques à I'hectare plus élevés que d'autres comme le centre du bassin parisien où les rendements en graines sont élevés ou comme la Bretagne et la Normandie où l'on consomme moins d'engrais azotés de synthèse, de par la valorisation des effluents d'élevage.

\section{Les caractéristiques de la production de biodiesel}

Pourquoi ces variations? Comment peut-on faire évoluer ces données dans un sens favorable?

En matière de bilan énergétique, l'énergie mobilisée au niveau de la production agricole, dépend essentiellement de trois grands postes:

- l'engrais azoté : I'engrais azoté coûte cher à produire d'un point de vue énergétique et constitue le premier poste de consommation énergétique pour des cultures comme le colza, le blé et dans une moindre mesure la betterave ;

- les consommations de fuel ;

- le matériel agricole : plus il est « lourd » plus sa production coûte en énergie.

Tableau 2. Rendement énergétique colza diester France. Variabilité entre années.

\begin{tabular}{|c|c|c|c|c|c|c|}
\hline Année & 1994 & 1996 & 1999 & 2001 & 2003 & 2005 \\
\hline Q5 & & 3,2 & 3,2 & 3,1 & 3,2 & 3,4 \\
\hline Médiane & 2,7 & 2,8 & 2,8 & 2,7 & 2,9 & 3,2 \\
\hline Q1 & & 2,7 & 2,7 & 2,5 & 2,7 & 3,0 \\
\hline
\end{tabular}

Source : Charte Colza - Cétiom. 
Tableau 3. Rendement énergétique colza diester. Variabilité de la moyenne.

\begin{tabular}{|c|c|c|c|c|c|c|}
\hline Régions & 1994 & 1996 & 1999 & 2001 & 2003 & 2005 \\
\hline Auvergne & & 2,9 & 2,9 & 2,9 & 2,7 & 3,1 \\
\hline Est & & 2,8 & 3,0 & 2,5 & 2,8 & 3,0 \\
\hline Nord & & 3,1 & 3,1 & 2,9 & 3,0 & 3,3 \\
\hline Nord-Est & & 2,9 & 3,0 & 2,5 & 2,9 & 3,1 \\
\hline Rhônes-Alpes & & 3,0 & 2,9 & & 2,8 & 3,2 \\
\hline Bretagne-Normandie & & 3,2 & 3,1 & 2,9 & 3,2 & 3,6 \\
\hline Centre & & 3,0 & 2,9 & 2,8 & 3,0 & 3,2 \\
\hline Ouest & & 3,1 & 3,0 & 3,0 & 3,0 & 3,2 \\
\hline Sud-Est & & 2,7 & 2,8 & 2,7 & 2,7 & \\
\hline Sud-Ouest & & 2,8 & 2,9 & & 2,8 & 3,0 \\
\hline
\end{tabular}

Source : Charte Colza - Cétiom.

L'irrigation compte peu dans ces exemples, du moins pour le colza, culture très rarement irriguée (tableau 4).

Une autre notion doit être prise en compte concernant les oléagineux est le fait que $n^{\prime} e s t$ utilisée que la partie huile de la graine (environ la moitié de la graine), si bien que les productions d'énergie à l'hectare reste moyenne.

Enfin, la comparaison de la production de Diester ${ }^{\circledR}$ à partir d'oléagineux et la production d'éthanol, souligne que la transformation des graines d'oléagineux (l'estérification) consomme relativement peu d'énergie par rapport à ce qui est consommé dans l'autre filière. Par contre, en termes de coût énergétique la production des graines oléagineuses est comparable à celui des autres filières, et si au final les performances de cette filière sont supérieures cela est dû essentiellement aux caractéristiques des process industriels qui suivent la production.

Le tableau 5 présente ainsi les résultats de l'étude Ademe Diren concernant les coûts énergétiques des deux oléagineux et des cultures de blé et de betteraves sucrières.

En termes de production agricole, l'énergie que I'on mobilise varie assez peu entre le colza et le blé, et l'énergie restituée y est pratiquement similaire. La betterave relativement peu fertilisée présentant des résultats les plus favorables.

\section{L'impact au niveau des gaz à effets de serre}

L'étude citée ci-dessus estime également la réduction des émissions de gaz à effet de serre de plus de 2,5 tonnes/ha de $\mathrm{CO}_{2}$. Les émissions de gaz à effet de serre des filières de biodiesel sont estimées à $24 \mathrm{~g}$ eq.CO2 (colza) et $20 \mathrm{~g}$ eq. $\mathrm{CO}_{2}$ (tournesol) par MJ de biocarburant, ce qui permet de diviser par 4 environ les émissions de gaz à effet de serre par rapport au diesel (tableau 6). En termes d'émission de gaz à effet de serre, ces filières $y$ contribuent principalement via le carbone (émissions de gaz carbonique) et via les émissions de protoxyde d'azote (notamment pour le colza).
Le gain net en termes d'émission des GES au niveau de l'hectare se traduit par le tableau 7.

\section{Comment peut-on analyser} et comprendre ces caractéristiques au niveau des oléagineux?

En termes de GES, 5 gaz doivent être pris en compte au niveau agricole :

- le $\mathrm{N}_{2} \mathrm{O}$, le protoxyde d'azote lié aux transformations de l'azote ;

- le $\mathrm{NH}_{3}$ l'ammoniac peu présent à l'échelle du champ agricole et plutôt lié à l'élevage ;

Tableau 4. Pratiques et rendement des cultures énergétiques pris en compte.

\begin{tabular}{|lcccc|}
\hline & Tournesol & Colza & Blé & Betterave sucrière \\
\hline Azote (unités) & 39 & 170 & 184 & 87 \\
Fuel (litre) & 177 & 201 & 107 & 184 \\
Rendement (q/ha) & 24,4 & 33,4 & 90,0 & 662 \\
\hline
\end{tabular}

Tableau 5. Energie mobilisée et énergie restituée par les cultures énergétiques.

\begin{tabular}{|lcccc|}
\hline GJ/ha & Tournesol & Colza & Blé & Betterave sucrière \\
\hline Azote & 1,9 & 8,9 & 9,7 & 5,1 \\
Fuel + mécanisation & 7,3 & 7,9 & 4,8 & 7,6 \\
Energie totale & 10,6 & 19,0 & 16,9 & 16,9 \\
mobilisée & & & & \\
Energie restituée & 79,7 & 108,8 & 97,9 & 125,2 \\
\hline
\end{tabular}

Tableau 6. Bilan des gaz à effet de serre (GES) des filières biocarburants complètes en 2005 avec I'hypothèse de combustion totale.

\begin{tabular}{|c|c|c|c|c|}
\hline Gazole & Huile de colza & Huile de tournesol & EMHV colza & EMHV tournesol \\
\hline 79,3 g.eqCO $2 / \mathrm{MJ}$ & 17,8 & 13,2 & 23,7 & 20,1 \\
\hline 3390 g.eqCO $2 / \mathrm{MJ}$ & 660 & 498 & 888 & 745 \\
\hline
\end{tabular}

Tableau 7. Gain net/ha des émissions des gaz à effet de serre (GES) pour quatre filières de biocarburants en 2005.

\begin{tabular}{|c|c|c|c|}
\hline & Rendements q/ha & Biocarburant produit (t/ha) & Gain teq $\mathrm{CO}_{2} /$ ha \\
\hline Colza & 33,4 & 1,37 & 3,46 \\
\hline Tournesol & 24,4 & 1,06 & 2,81 \\
\hline Blé & 90,0 & 2,55 & 6,95 \\
\hline Betteraves & 662,4 & 5,78 & 15,88 \\
\hline
\end{tabular}


Autre élément important, aujourd'hui les résidus de culture les pailles de colza et de tournesol sont systématiquement enfouis ce qui contribue à améliorer les stocks de carbone dans le sol.

Enfin, on estime que le travail simplifié pourrait contribuer à stocker du carbone sous réserve que le labour avec retournement ne soit jamais utilisé : actuellement, une parcelle sur deux de colza n'est pas labourée pour son implantation, chiffre qui a vite augmenté ces dernières années. En tournesol, la progression plus lente avec seulement une parcelle sur cinq qui $n^{\prime}$ est pas travaillée.

D'un point de vue coût énergétique, cette situation est intéressante : on réduit ainsi les consommations en fuel. Ceci dit est-ce positif du point de vue du stockage du carbone ? Tout dépend des modalités de travail du sol dans la durée. Si dans la rotation on laboure toutes les cultures sauf les oléagineux, il est probable qu'on fait peu évoluer le stock de carbone dans le sol : si la simplification du travail n'est que ponctuelle dans la rotation il est probable que cela ne permet pas de gagner beaucoup par contre si ce non-travail, ce non-labour est permanent, là il semble que l'on ait de vrais gains à envisager.

\section{Les tendances d'évolution de la production agricole de graines oléagineuses}

\section{Le colza}

Depuis les années 2000, pour la production de colza on constate une forte progression de la sole en surface, progression qui se fait pour l'essentiel hors des zones traditionnelles. Historiquement le colza était cultivé en effet dans le croissant des terres à cailloux de l'Est et du Sud du bassin parisien. Avec le développement du colza énergétique depuis de dix ans le colza est « sorti » de ses zones traditionnelles et se développer notamment dans le centre du Bassin parisien.

Ainsi, il y a 20 ans, le principal oléagineux en Eure-et-Loir, en pleine Beauce, était le tournesol : aujourd'hui il n'y a pratiquement plus de tournesol dans ce département et le premier département producteur de colza en surface et en rendement, n'est plus en Lorraine, en Bourgogne, ni en Champagne berrichonne, mais le département d'Eure-et-Loir.

On cultive ainsi de plus en plus de colza dans des sols limoneux et profonds qu'il y a une vingtaine d'années. Or, le colza a un potentiel de rendement très élevé dans ces sols. C'est pourquoi il faut relativiser la part de l'amélioration du matériel génétique et des techniques de production dans la progression des rendements moyens nationaux au profit de cette progression des surfaces dans des zones à plus fort potentiel.

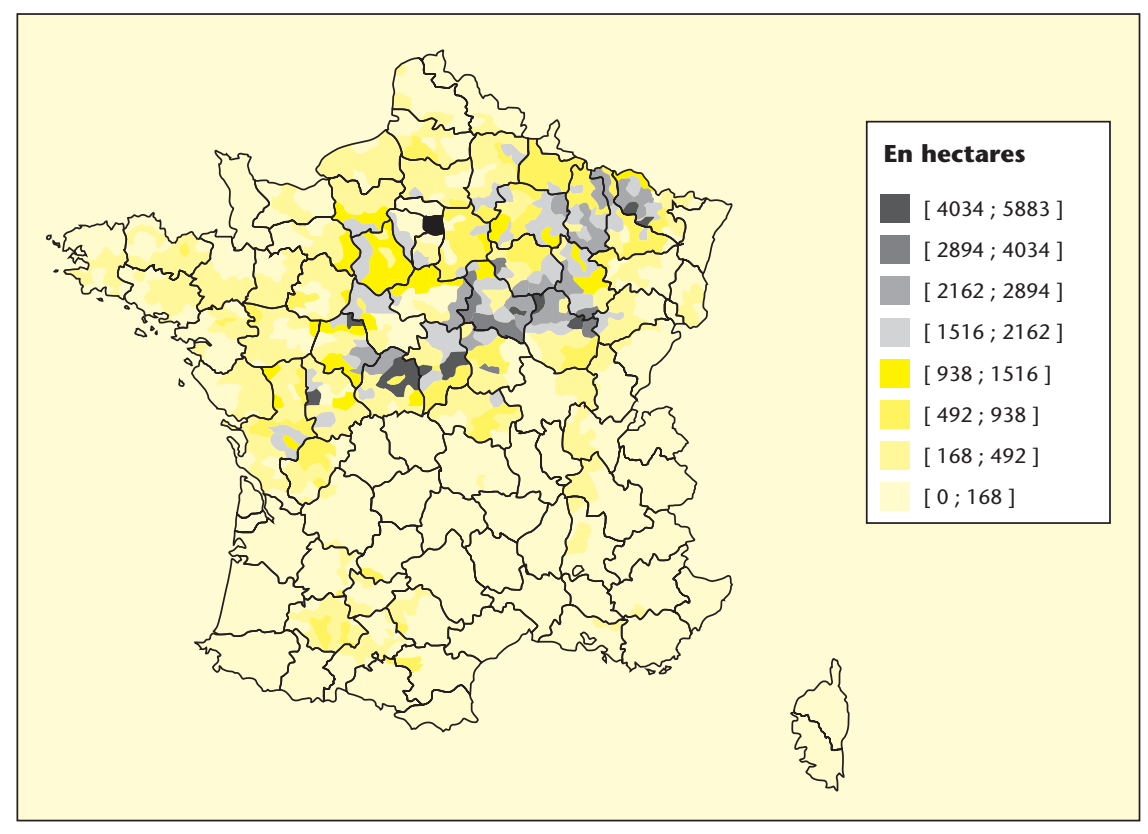

Figure 1. Les surfaces cultivées en colza (hiver, printemps). Par canton (2000).

La carte des superficies en France (figure 1) montre que dès l'année 2000 le colza était présent au centre du Bassin parisien et on sait qu'actuellement il est en train de se développer plus au nord et notamment en Picardie. Ce qui contribue fortement à l'augmentation des rendements : on peut obtenir des résultats approchant les $40 \mathrm{q} / \mathrm{ha}$ au centre du Bassin parisien quand il est beaucoup plus difficile de les faire dans les petites terres à cailloux, notamment les années où le climat est relativement sec.

Depuis dix ans, on assiste également à une baisse de la fertilisation azotée notamment suite aux travaux du Cétiom sur le raisonnement de la fertilisation azotée et le développement de différents outils de raisonnement.

Pour illustrer les doses d'engrais appliquées sur colza et issues des enquêtes Cetiom sur les pratiques culturales de colza et réalisées une année sur deux depuis le début des années 90 : il semble que l'on ait une baisse régulière des doses d'azote appliquée sur le colza (figure 2). Soit une baisse de 10 unités depuis une dizaine d'années.

Du côté de l'industrie des engrais on constate également une nette amélioration du bilan énergétique de la fabrication des engrais azotés. Au moins au niveau de l'Ouest européen, avec des processus comme la cogénération on commence à récupérer l'énergie des réactions chimiques les plus exothermiques ce qui fait que I'Unifa ${ }^{1}$ affirme actuellement que si il y a

${ }^{1}$ UNIFA : Union nationale interprofessionnelle des fabricants d'engrais et d'amendements. quelques années les références de coût énergétique étaient de l'ordre de $50 \mathrm{M}$ / unité, ces coûts sont déjà réduits de $20 \%$. Ces indications laissent espérer une contribution de l'industrie des engrais nous permettant d'améliorer ces bilans énergétiques.

Enfin dernier point, il y a un fort développement du travail simplifié, ce qui est favorable à la réduction des coûts énergétiques de production des graines oléagineuses.

La figure 3 montre l'évolution des rendements moyens nationaux : globalement sur dix ans le colza est en nette progression en tendance.

\section{Le tournesol}

Globalement, si la sole de colza s'étend, la sole de tournesol se réduit en se concentrant vers le sud-ouest et le centre ouest, deux régions où il n'y a pas de recul des surfaces. Dans le SudOuest par exemple, il est cultivé une année sur deux dans la moitié des parcelles c'est-à-dire dans le cadre de rotation courte (ce qui expose la culture à plus de maladie ou d'attaques de ravageurs que dans des rotations plus longues). Cette réduction des surfaces est ainsi négative d'un double point de vue, car le potentiel du rendement est plus faible en rotation courte comme il est plus faible sous le climat séchant du sud de la France ( figure 4).

Le tournesol demande une fertilisation azotée peu élevée. On bénéficie ainsi des coûts énergétiques modérés de par la baisse du coût des engrais. L'azote représente une part relativement faible en termes d'énergie mobilisée dans cet écobilan.

Par contre la simplification du travail du sol reste limitée pour le tournesol. 


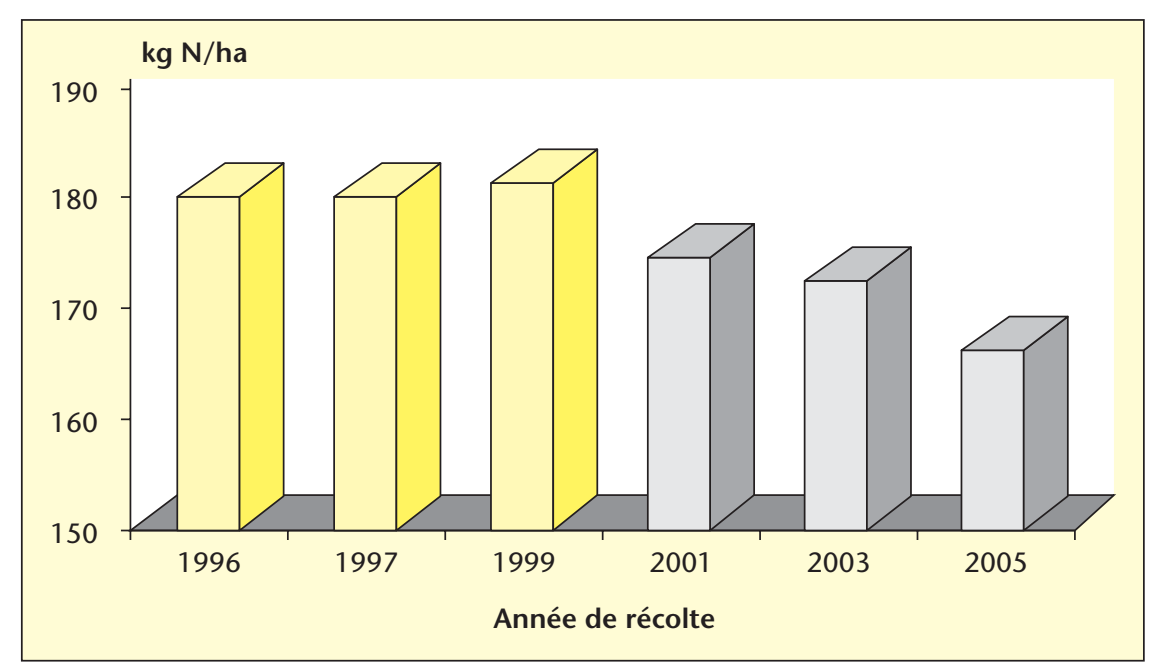

Figure 2. Engrais azoté pour le colza : des doses en baisse. Source : Charte Colza - Cétiom.

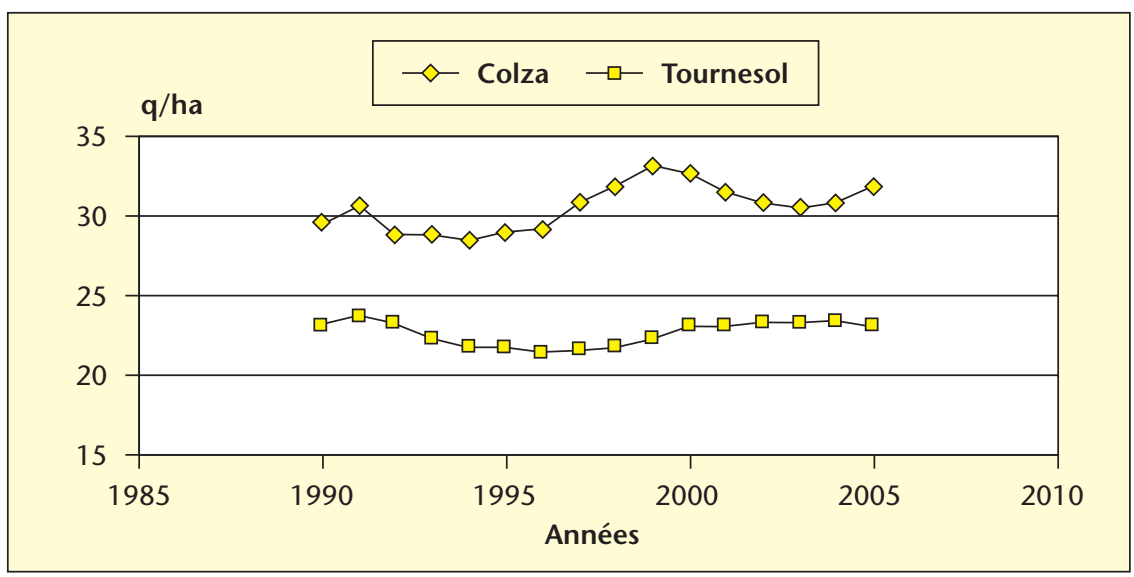

Figure 3. Progression des rendements du colza. Rendements moyen glissant sur 5 ans. Source : Scees.

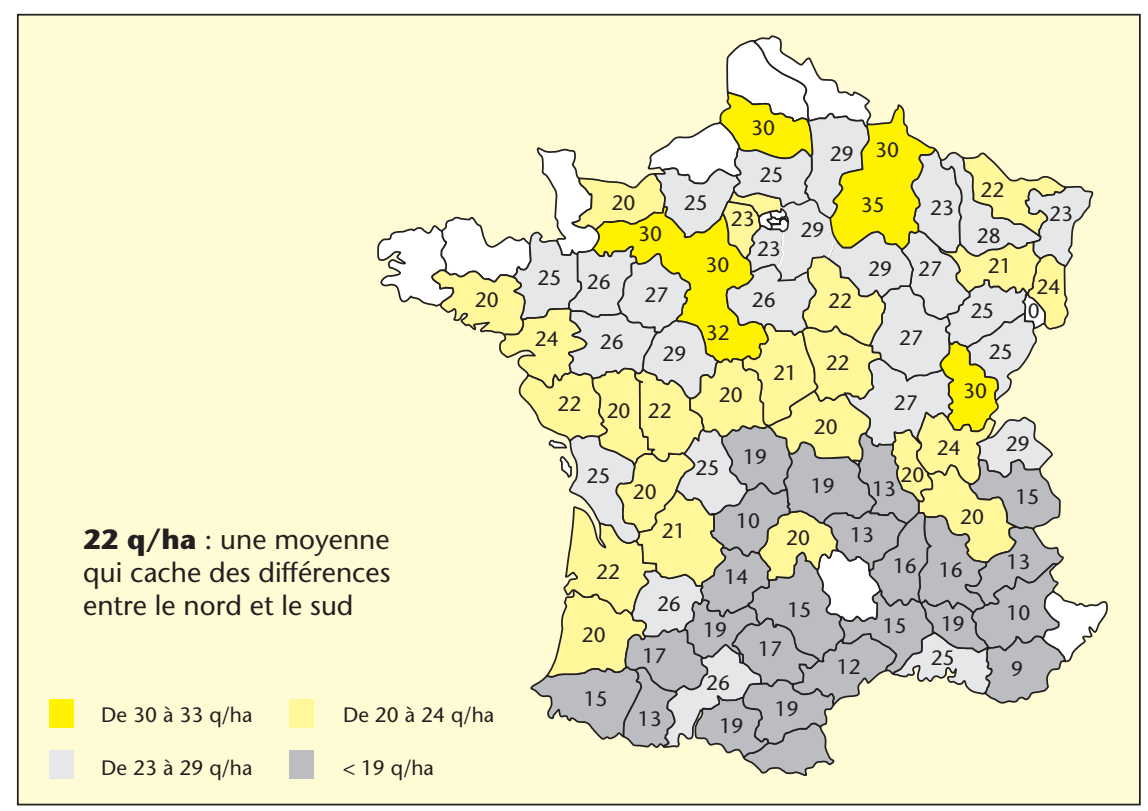

Figure 4. Carte des rendements du tournesol en 2003. Source : Scees.

\section{Quelles sont les perspectives de demain?}

Ce qui était inscrit dans l'étude Ademe-Direm se réalise actuellement assez bien tant en matière de fertilisation azotée, que de rendement et de travail du sol.

Quels autres changements pourrait-on avoir à terme?

La génétique du colza laisse entrevoir des perspectives d'évolution. Développer la richesse en huile des graines, réduire la teneur en azote des résidus de colza (pailles et feuilles), pourrait permettre de progresser tant du point de vue des pertes en azote, (nitrate et protoxyde d'azote), sur le stockage de carbone dans le sol, comme en réduction des besoins en engrais azotés, un poste important par rapport à l'énergie mobilisée dans cette filière.

D’autres évolutions pourraient infléchir ces performances. Le tournesol va-t-il continuer à se cantonner à ses deux grandes régions traditionnelles ou pourra-t-il revenir dans d'autres régions? Jusqu'où va aller le développement du colza dans les sols limoneux et profonds? Deux questions importantes dans l'évolution des performances des cultures pour les biocarburants.

Comment vont progresser les cultures intermédiaires utiles avant le tournesol ou après le colza. C'est un sujet important du point de vue du stockage du carbone ? Est-ce que l'irrigation du tournesol va rester à un niveau très faible ou va-t-elle finir par se développer? C'est une autre question. Enfin la co-génération et la valorisation des pailles sont de plus en plus d'actualité : la valorisation des pailles va-t-elle se limiter uniquement aux céréales ou va-t-elle concerner également les pailles de colza : c'est une autre question.

\section{Conclusion}

Pour bien évaluer ces perspectives nous avons besoin de réaliser des diagnostics sur l'évolution des pratiques et de développer des outils d'aide à la décision. Les centres techniques comme le Cetiom et l'Iterg y participent activement pour les filières biocarburants à partir d'oléagineux.

Dans le cadre de ce développement des filières colza et tournesol Diester, le Cetiom met maintenant en place une seconde Charte oléagineux pour aider les agriculteurs et leurs conseillers à cerner comment mieux conduire le colza et le tournesol pour améliorer la contribution des biocarburants au développement durable. 